\title{
Roaming dynamics in ketene isomerization
}

\author{
Frédéric A. L. Mauguière • Peter Collins • \\ Gregory S. Ezra $\cdot$ Stavros C. Farantos $\cdot$ \\ Stephen Wiggins
}

Received: 25 March 2014/Accepted: 19 May 2014/Published online: 10 June 2014

(C) The Author(s) 2014. This article is published with open access at Springerlink.com

\begin{abstract}
A reduced two-dimensional model is used to study ketene isomerization reaction. In light of recent results by Ulusoy et al. (J Phys Chem A 117, 7553, 2013), the present work focuses on the generalization of the roaming mechanism to the ketene isomerization reaction by applying our phase space approach previously used to elucidate the roaming phenomenon in ion-molecule reactions. Roaming is again found be associated with the trapping of trajectories in a phase space region between two dividing surfaces; trajectories are classified as reactive or nonreactive, and are further naturally classified as direct
\end{abstract}

Dedicated to Professor Greg Ezra and published as part of the special collection of articles celebrating his 60th birthday.

F. A. L. Mauguière · P. Collins · S. Wiggins ( $\square)$

School of Mathematics, University of Bristol,

Bristol BS8 1TW, UK

e-mail: s.wiggins@bristol.ac.uk

F. A. L. Mauguière

e-mail: frederic.mauguiere@bristol.ac.uk

P. Collins

e-mail: Peter.Collins@bristol.ac.uk

G. S. Ezra

Baker Laboratory, Department of Chemistry and Chemical

Biology, Cornell University, Ithaca, NY 14853, USA

e-mail: gse1@ cornell.edu

\section{S. C. Farantos}

Institute of Electronic Structure and Laser, Foundation

for Research and Technology - Hellas, Iráklion,

Crete 711 10, Greece

e-mail: farantos@iesl.forth.gr

S. C. Farantos

Department of Chemistry, University of Crete, Iráklion,

Crete 711 10, Greece or nondirect (roaming). The latter long-lived trajectories are trapped in the region of nonlinear mechanical resonances, which in turn define alternative reaction pathways in phase space. It is demonstrated that resonances associated with periodic orbits provide a dynamical explanation of the quantum mechanical resonances found in the isomerization rate constant calculations by Gezelter and Miller (J Chem Phys 103, 7868-7876, 1995). Evidence of the trapping of trajectories by 'sticky' resonant periodic orbits is provided by plotting Poincaré surfaces of section, and a gap time analysis is carried out in order to investigate the statistical assumption inherent in transition state theory for ketene isomerization.

Keywords Roaming reaction - Normally hyperbolic invariant manifold - Periodic orbit - Nonlinear resonance . Transition state and dividing surface $\cdot$ Gap time distribution

\section{Introduction}

The photodissociation of ketene, $\mathrm{CH}_{2} \mathrm{CO}$, has been the subject of many studies, both experimental and theoretical. Moore and co-workers, in a series of experiments, have reported many interesting aspects of this reaction [1-3]. The dissociation gives rise to two product fragments, $\mathrm{CO}$ and $\mathrm{CH}_{2}$. However, when the dissociation is observed in molecules for which the two carbons are differentiated by using different isotopes, with for example, ${ }^{12} \mathrm{CH}_{2}^{13} \mathrm{CO}$ or ${ }^{13} \mathrm{CH}_{2}^{12} \mathrm{CO}$, then the products of the dissociation show in each case a mixture of ${ }^{13} \mathrm{CO}$ and ${ }^{12} \mathrm{CO}$ with co-fragments ${ }^{13} \mathrm{CH}_{2}$ and ${ }^{12} \mathrm{CH}_{2}$. These results show that isomerization (carbon exchange) in ketene has taken place prior to dissociation. The postulated mechanism involved in the 
isomerization of ketene is the Wolff rearrangement mechanism [4]. In order to examine this hypothesis, $\mathrm{ab}$ initio calculations have been carried out by several authors $[1,5]$. These calculations showed that the relevant portion of the potential energy surface (PES) for ketene isomerization has three different minima associated with two symmetrically related formylmethylene species and an oxirene structure situated midway between these structures, respectively [3]. On each side, a high barrier leads to the two isomers of ketene. These findings point out the importance of understanding the isomerization of ketene when studying its dissociation.

Recently, Ulusoy et al. [6, 7] studied the effect of roaming trajectories on the reaction rates for the isomerization of ketene. The roaming phenomenon is a recently identified chemical reaction mechanism, which has attracted much attention due to its unusual character. The roaming phenomenon was discovered in the photodissociation of $\mathrm{H}_{2} \mathrm{CO}$ [8]. In this process, the formaldehyde molecule can dissociate via two channels: $\mathrm{H}_{2} \mathrm{CO} \rightarrow \mathrm{H}+$ $\mathrm{HCO}$ (radical channel) and $\mathrm{H}_{2} \mathrm{CO} \rightarrow \mathrm{H}_{2}+\mathrm{CO}$ (molecular channel). Zee et al. [9] pointed out that, above the threshold for the $\mathrm{H}+\mathrm{HCO}$ dissociation channel, the $\mathrm{CO}$ rotational state distribution exhibited an intriguing "shoulder" at lower rotational levels correlated with a hot vibrational distribution of $\mathrm{H}_{2}$ co-product. The observed product state distribution did not fit well with the traditional picture of the dissociation of formaldehyde via a well-characterized saddle point transition state for the molecular channel. The roaming mechanism as an alternative reaction pathway, which explains the observations of van Zee and co-workers, was demonstrated both experimentally and in trajectory simulations by Townsend et al. [8]. Following this work, roaming has been identified in the unimolecular dissociation of molecules such as $\mathrm{CH}_{3} \mathrm{CHO}, \mathrm{CH}_{3} \mathrm{OOH}$ or $\mathrm{CH}_{3} \mathrm{CCH}$, as well as in ion-molecule reactions [10], and it is now recognized as a general phenomenon in reaction dynamics (see refs $[11,12]$ and references therein).

A common characteristic of systems exhibiting roaming reactions studied so far is the presence of long-range interactions between the fragments of the dissociating molecule. However, the study by Ulusoy et al. [6, 7] discussed the roaming phenomenon in a different context. For ketene isomerization, there are no long-range interactions at play and this raises the question of the broader relevance of the roaming mechanism. Ulusoy et al. [6, 7] in their effort to find trajectories that avoid the minimum energy path (MEP) on the potential energy surface carried out isomerization rate constant calculations at very high energies, accessible neither to experiments [1-3] nor to the quantum mechanical calculations of Gezelter and Miller [13].

In two recent studies [14, 15], we have analysed the roaming phenomenon for ion-molecule reactions. In such reactions, the long-range potential function is dominated by charge-induced dipole interactions; a useful model for these systems is the Chesnavich potential [16]. By investigating the dynamics in its appropriate setting, phase space, we examined the roaming phenomenon in the presence of well-defined dividing surfaces (DSs) and associated reaction pathways in phase space. This approach enabled us to interpret roaming as a trapping phenomenon of trajectories between two DSs and the enhancement of this trapping by the presence of nonlinear mechanical resonances between the different degrees of freedom (DoF) of the system. ketene offers the opportunity to investigate the roaming phenomenon in other type of molecules than those studied up to now, and this is the main purpose of the present article.

The paper is organized as follows. Section 2 presents the reduced dimensional model for ketene isomerization used in our study and originally introduced by Gezelter and Miller [13]. We then analyse the dynamics of ketene isomerization in Sect. 3. In this section, we first discuss the construction of DSs that govern the reactions studied in Sect. 3.1 and then the dynamics in Sect. 3.2. The next Sect. 3.3 investigates the statistical assumption for the dynamics by a gap time analysis. Sect. 3.4 presents a detailed analysis of the trapping mechanism of trajectories between the DSs and the role of the ubiquitous resonances due to bifurcations of periodic orbits. In Sect. 4, roaming observed in the two distinctly different systems, ion-molecule and ketene, is compared. By associating roaming to the phase space structure of the molecule in a dynamically well-defined fashion, we establish its general character as a framework for revealing and exploring new reaction pathways. Finally, Sect. 5 concludes.

\section{Reduced dimensional model of ketene isomerization}

In this section, we give the details of the Hamiltonian used in our study. A reduced dimensional model for the study of ketene isomerization was proposed several years ago by Gezelter and Miller [13]. In their original paper, they proposed three models for ketene isomerization, retaining one, two and three relevant DoF for describing the isomerization process of ketene amongst the nine DoF of ketene molecule, respectively. They subsequently employed these three models for calculating quantum mechanical isomerization reaction rates. From their study, it appears that a two-dimensional model provides the best agreement when compared with experimental results by Lovejoy et al. [3]. Ulusoy et al. employed this two DoF model in their classical mechanical study $[6,7]$ of the dynamics of isomerization of ketene, and we use the same model in the present study. 
In the Gezelter and Miller model, one of two DoF is identified with the reactive mode and involves mainly oxygen and hydrogen atom motion. The other relevant DoF corresponds to the out-of-plane motion of a hydrogen atom relative to the $\mathrm{CCO}$ plane, a motion which has a harmonic frequency of approximately $514 \mathrm{~cm}^{-1}$. Gezelter and Miller used an analytical form for their reduced dimensional potential having several adjustable parameters in it. They fitted these parameters to reproduce the data from $a b$ initio calculations obtained by Scott et al. [5]. The resulting potential $^{1}$ is expressed as:

$$
V\left(q_{F}, q_{1}\right)=V_{1 d}\left(q_{F}\right)+\frac{1}{2} k\left(q_{1}+\frac{d}{k} q_{F}^{4}\right)^{2},
$$

with

$$
V_{1 d}\left(q_{F}\right)=a_{2} q_{F}^{2}+a_{4} q_{F}^{4}+a_{6} q_{F}^{6}+c q_{F}^{2} \exp \left(-d_{0} q_{F}^{2}\right) .
$$

Numerical values of the potential parameters are given in Table1. Figure 1 depicts a contour plot of the potential along with some periodic orbits (see Sect. 3.4). The different equilibrium points (EP) of the potential are listed in Table 2. For each EP, the stability is indicated by the labels CC or CS. A CC label denotes a centre-centre EP, which means that the EP is stable in both directions and the label CS is used for centre-saddle, which means that the EP is stable in one direction and unstable in the other.

The total Hamiltonian is obtained by adding to the above potential a kinetic energy term quadratic in momenta $\left(p_{q_{F}}, p_{q_{1}}\right)$ conjugate to the coordinates $\left(q_{F}, q_{1}\right)$, respectively. The resulting Hamiltonian takes the form:

$H\left(p_{q_{F}}, p_{q_{1}}, q_{F}, q_{1}\right)=\frac{p_{q_{F}}^{2}}{2 m_{\mathrm{O}}}+\frac{p_{q_{1}}^{2}}{2 m_{\mathrm{H}}}+V\left(q_{F}, q_{1}\right)$,

where $m_{\mathrm{O}}=16 \mathrm{u}$ and $m_{\mathrm{H}}=1 \mathrm{u}$ are the masses of oxygen and hydrogen atom, respectively. This choice of masses was used by Gezelter and Miller [17].

\section{Dynamics of ketene isomerization}

In this work, we are concerned with the dynamics of isomerization in ketene and its relation to the roaming phenomenon. Recently, Ulusoy et al. reported a study of ketene isomerization where they analysed the impact of roaming trajectories on the rate of the reaction $[6,7]$. Up to now, roaming reactions have been identified in the context of reactions involving long-range interactions between two fragments of a dissociating molecule. The study of Ulusoy

\footnotetext{
${ }^{1}$ Note that, the original paper by Gezelter and Miller [13] had a typo in it resulting in a missing factor of one half in front of the second term in the RHS of their Eq. (2.2).
}

Table 1 Parameters used in the reduced dimensional potential of ketene

\begin{tabular}{lll}
\hline Parameter & Value & Units \\
\hline$k$ & $1.0074 \times 10^{-2}$ & $\left(E_{h} a_{0}^{2}\right)$ \\
$d$ & 1.9769 & $\left(E_{h} a_{0}^{5}\right)$ \\
$a_{2}$ & $-2.3597 \times 10^{-3}$ & $\left(E_{h} a_{0}^{2}\right)$ \\
$a_{4}$ & $1.0408 \times 10^{-3}$ & $\left(E_{h} a_{0}^{4}\right)$ \\
$a_{6}$ & $-7.5496 \times 10^{-5}$ & $\left(E_{h} a_{0}^{6}\right)$ \\
$c$ & $7.7569 \times 10^{-3}$ & $\left(E_{h} a_{0}^{2}\right)$ \\
$d_{0}$ & $-2.45182 \times 10^{-4}$ & $\left(a_{0}^{2}\right)$ \\
\hline
\end{tabular}

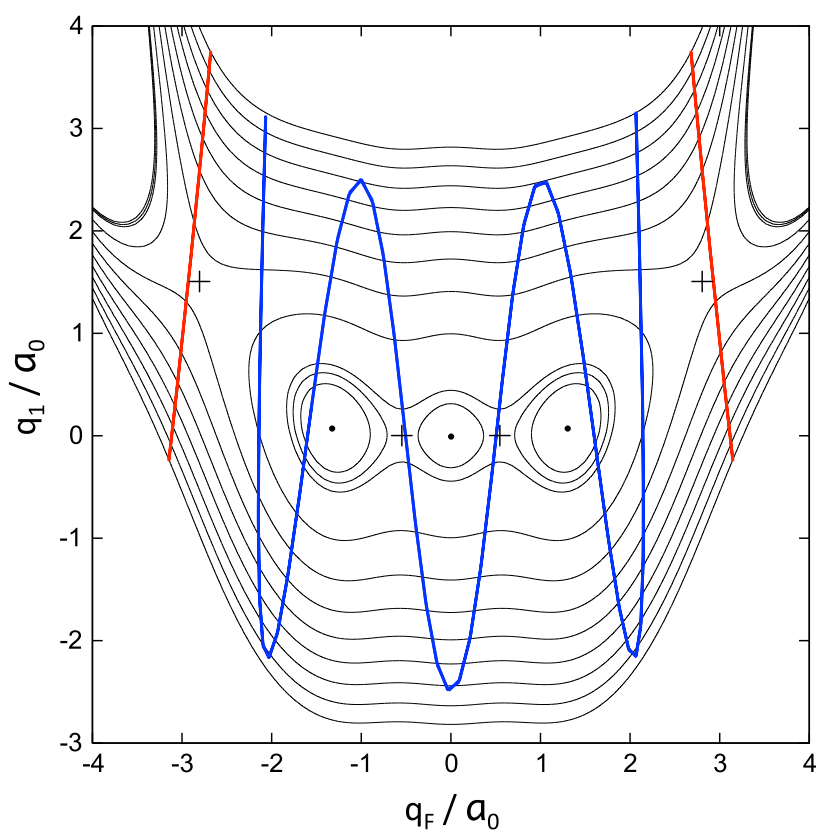

Fig. 1 Contour plot of the PES along with some POs. The red curves are the Lyapunov POs, and the blue is a 1:6 resonant PO (see text)

Table 2 Equilibrium points of the potential with their stability

\begin{tabular}{lcll}
\hline Name & $q_{\mathrm{F}}$ & $q_{1}$ & Stability \\
\hline $\mathrm{EP}_{1}$ & -2.805 & 1.506 & $\mathrm{CS}$ \\
$\mathrm{EP}_{2}$ & -1.325 & 0.075 & $\mathrm{CC}$ \\
$\mathrm{EP}_{3}$ & -0.547 & 0.002 & $\mathrm{CS}$ \\
$\mathrm{EP}_{4}$ & 0.0 & 0.0 & $\mathrm{CC}$ \\
$\mathrm{EP}_{5}$ & 0.547 & 0.002 & $\mathrm{CS}$ \\
$\mathrm{EP}_{6}$ & 1.325 & 0.075 & $\mathrm{CC}$ \\
$\mathrm{EP}_{7}$ & 2.805 & 1.506 & $\mathrm{CS}$ \\
\hline $\mathrm{CoO}$
\end{tabular}

Coordinates $q_{F}$ and $q_{1}$ are indicated in Bohr. CS means centre-saddle and CC centre-centre

et al. suggests the possibility of looking at roaming in different reaction dynamics contexts and in this sense represents an important conceptual advance. Recently, we 
studied the dynamics of roaming reactions in the context of ion-molecule reactions $[14,15]$ and proposed a dynamical origin for the roaming phenomenon. Our approach consisted of identifying optimal DSs for the different reactive events possibly occurring in the problem considered. The roaming phenomenon was then identified with the trapping of trajectories between two DSs and enhanced by the presence of nonlinear resonances identified with resonant periodic orbits (POs). In the present work, we extend our methods to study the ketene isomerization reaction and the way roaming appears. To begin, we must first locate the relevant DSs for the problem we consider. The next paragraph is devoted to the definition of proper DSs and how this can be achieved with the help of the so-called normally hyperbolic invariant manifolds (NHIMs).

\subsection{Normally hyperbolic invariant manifolds and dividing surfaces}

The study of reaction dynamics aims at understanding the associated mechanism at an atomic level of detail in a specific molecular reaction. Eventually, one is interested in computing quantities such as reaction rate constants, which can be compared with experiment. The most popular theory developed for this purpose is transition state theory (TST). TST relies on several fundamental assumptions [18]. One of these assumptions is the existence of a dividing surface, which separates reactants from products and which has the nonrecrossing property. This means that a trajectory initiated at the reactants and crossing this dividing surface must proceed to products without recrossing the dividing surface, and end up as a reactant. The other fundamental assumption of TST is that of statistical dynamics. If one considers the reaction at a specific energy (microcanonical), the statistical assumption requires that throughout the dissociation of the molecule, all phase space points are equally probable on the timescale of reaction [18]. This assumption is equivalent to saying that the redistribution of the energy amongst the different DoF of the system on the reactant side of the DS is fast compared to the rate of the reaction, and this guarantees a single exponential decay for the reaction. We will check this assumption for the ketene model by a gap time analysis in the roaming region (see below).

In our previous study of roaming phenomena, we explained how NHIMs provide a solution to the problem of the nonrecrossing property of the DS. In fact, the problem of constructing a nonrecrossing DS for a two DoF system was solved during the 1970s by Pechukas, Pollak and coworkers [19-22]. They showed that the DS at a specific energy is intimately related to an invariant phase space object, an unstable PO. The periodic orbit defines the bottleneck in phase space through which the reaction occurs, and the DS which intersects trajectories evolving from reactants to products can be shown to have the topology of a hemisphere, whose boundary is the PO. As our present system is a two DoF problem, we will focus on POs and the DSs constructed from them. Nevertheless, this unstable PO is a simple example of a NHIM. Generalization to higher number of DoF has been a major obstacle in the development of the theory, and the question of the construction of DSs for systems with three and more DoF has been given a satisfactory answer only recently. The appropriate construction is built around an invariant phase space object, a NHIM, which is the generalization of the unstable PO of the two DoF case. The NHIM serves as the anchor for the construction of the DS, and this DS constructed in this way can be shown to have the nonrecrossing property [23].

The construction of a nonrecrossing DS for the simple two DoF model of ketene isomerization therefore starts with the location of relevant unstable POs. For the ketene problem, isomerization reaction is completed when the system passes from one ketene well to the other. The model we are dealing with locates the oxirene minimum between the formylmethylene wells. If we consider that the system is initially in the ketene well located on the part of the potential for which $q_{F}<-3 a_{0}$ (the "left' ketene well if we look at Fig. 1), then isomerization is completed if the system passes to the other ketene well located on the part of the potential for which $q_{F}>3 a_{0}$. To accomplish this reaction, we see that the system must pass through two bottlenecks located around the equilibria labelled $\mathrm{EP}_{1}$ and $\mathrm{EP}_{7}$ in Table 2. These two EPs are of CS stability type, which means that the linearized vector field at these points (with momenta $p_{q_{F}}=0$ and $p_{q_{1}}=0$ ) has one pair of real eigenvalues and one pair of imaginary eigenvalues. This means that in the vicinity of these EPs, there exist unstable POs [24], the so-called Lyapunov POs [25]. In the vicinity of these EPs, the Lyapunov POs exist as families of POs depending on the energy of the system. Representative POs of these families are shown in Fig. 1. These POs are the NHIMs which define the bottlenecks through which the reaction occurs and will serve as anchors for the construction of the nonrecrossing DSs.

The DSs attached to these NHIMs are obtained by considering a fixed value of energy. At each energy, two Lyapunov POs exist, each located in the vicinity of $\mathrm{EP}_{1}$ and $\mathrm{EP}_{7}$, respectively. These POs are the equators of the DSs [23] and split the DSs into two hemispheres. One hemisphere intersects trajectories travelling from reactant to products (forward hemisphere) and the other intersects trajectories travelling from products to reactants (backward hemisphere). For concreteness, we consider the Lyapunov 
$\mathrm{PO}$ at a certain energy in the vicinity of $\mathrm{EP}_{1}$. This $\mathrm{PO}$ projects to a line in configuration space (see Fig. 1). To sample points on the DS, we need to specify the phase space coordinates of the points belonging to the DS at a certain energy. To do so, we move along the PO (the equator of the DS) in configuration space. This fixes the values of $q_{F}$ and $q_{1}$. We then scan the values of one of the momenta $p_{q_{F}}$ or $p_{q_{1}}$. To obtain the value of the remaining phase space coordinate (the remaining momentum), we solve the equation $H\left(p_{q_{F}}, p_{q_{1}}, q_{F}, q_{1}\right)=E$ for the remaining unknown momentum $\left(p_{q_{F}}\right.$ or $\left.p_{q_{1}}\right)$ and at a specified value of energy, $E$. Since the kinetic energy is quadratic in the momenta, this equation has two real roots distinguished by their sign. Each root belongs to one of the hemispheres of the DS (forward and backward hemispheres). The result of this sampling procedure is shown in Fig. 2. Panel (a) of this figure is a 3D plot of the forward hemisphere (blue dots) of the DS at energy of 0.01 Hartree above the oxirene minimum. The Lyapunov PO is shown by the green curve. Panel (b) depicts a 2D projection of this hemisphere onto the $\left(q_{1}, p_{q_{1}}\right)$ plane. At the bottom of this figure, we note that some points belonging to the hemisphere lie out of the area enclosed by the Lyapunov PO (green curve). This is the result of the 'tilting' over of the DS in the coordinates we are using. Because of this fact, to obtain a uniform sampling with full coverage of the hemisphere, we sample momenta in two ways: sampling $p_{q_{1}}$ with $p_{q_{F}}$ obtained by solving the energy equation mentioned above, or sampling $p_{q_{F}}$ and fixing $p_{q_{1}}$ from the equation of energy.

\subsection{Classical trajectory simulations}

Our aim is to investigate the dynamics of ketene isomerization and how one can identify and understand the roaming phenomenon in this situation. To do so, we propagated classical trajectories and examined their qualitatively different characters.

If we consider that the system is initially located in the ketene well in the region where $q_{F}<-3 a_{0}$, then the isomerization reaction is completed when the system arrives in the ketene well on the region where $q_{F}>3 a_{0}$. On its way to complete this reaction, a trajectory has to pass through two bottlenecks, one located in the vicinity of $\mathrm{EP}_{1}$ and which is defined by the Lyapunov $\mathrm{PO}$ we discussed above, and the other located in the vicinity of $\mathrm{EP}_{7}$ also defined by a Lyapunov PO. We explained above how to construct DSs attached to these two POs. In order to understand the dynamics of the isomerization process, we sampled the DS attached to the Lyapunov PO associated with $\mathrm{EP}_{1}$. We used these samples as initial conditions for numerically propagating classical trajectories.

As we did in our previous studies [14, 15] of roaming phenomenon, we classified the trajectories into four qualitatively different categories. These different categories of trajectories are defined in relation to the reactive scenario involved in the ketene isomerization. To begin, we note that there are two obvious different types of expected trajectories. First, there are reactive trajectories which will complete the isomerization reaction, which means that these type of trajectories will cross the DS located in the vicinity of $\mathrm{EP}_{7}$. Second, there are nonreactive trajectories, which do not complete the isomerization reaction, but instead recross the DS located in the vicinity of $\mathrm{EP}_{1}$ and end up in the ketene well where they originally came from.

Since we are interested in understanding the roaming phenomenon from a dynamical point of view, we need to specify those characteristics a trajectory should have to be assigned as roaming. The notion of roaming is invoked when a trajectory does not follow the minimum energy path (MEP) or intrinsic reaction coordinate (IRC) [26] of the potential function. In our problem, this departure from traditional understanding of reactivity occurs in trajectories, which do not complete directly the isomerization reaction or do not recross directly the DS located in the vicinity of $\mathrm{EP}_{1}$. Instead, the roaming trajectories spend more time in the trapping (roaming) region and exhibit oscillations in the $q_{F}$ direction before eventually finding their way out of the oxirene well region by either crossing
Fig. 2 Forward hemisphere of the dividing surface at the top left saddle at energy $E=0.01$ $E_{h}$. a 3-D view. b Projection on the $\left(q_{1}, p_{q_{1}}\right)$ plane
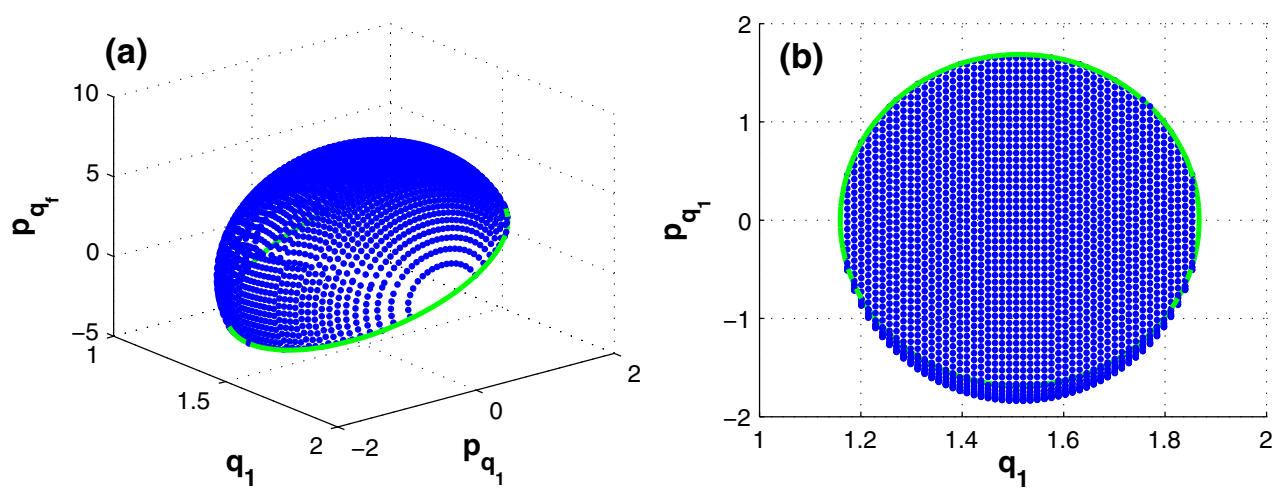
the DS located in the vicinity of $\mathrm{EP}_{7}$ or the DS located in the vicinity of $\mathrm{EP}_{1}$. To summarize, reactive (which complete the isomerization reaction) and nonreactive (which do not complete isomerization) trajectories are further categorized as direct and roaming. Hence, we have four classes of trajectories: direct reactive, roaming reactive, direct nonreactive and roaming nonreactive. However, in order to quantitatively define a roaming trajectory, we need a criterion to decide when a trajectory exhibits oscillations in the $q_{F}$ direction before eventually becoming reacting or nonreacting. To achieve this task, we count the number of times a trajectory crosses the symmetry line $q_{F}=0$. The precise definitions of the four categories are therefore:

1. Direct reactive trajectories: these trajectories cross the line $q_{F}=0$ only once before crossing the DS located in the vicinity of $\mathrm{EP}_{7}$ and completing the isomerization reaction.

2. Roaming reactive trajectories: these trajectories cross the line $q_{F}=0$ at least three times before crossing the DS located in the vicinity of $\mathrm{EP}_{7}$ and complete the isomerization reaction. Note that a reactive trajectory has to cross the line $q_{F}=0$ an odd number of times.

3. Direct nonreactive trajectories: these trajectories cross the line $q_{F}=0$ only twice before crossing the DS located in the vicinity of $\mathrm{EP}_{1}$ and do not complete the isomerization reaction.

4. Roaming nonreactive trajectories: these trajectories cross the line $q_{F}=0$ at least four times before crossing the DS located in the vicinity of $\mathrm{EP}_{1}$ and do not complete the isomerization reaction. Note that nonreactive trajectories have to cross the line $q_{F}=0$ an even number of times.
In Fig. 3, we show representative trajectories from these four categories. Each panel in this figure corresponds to a particular category. Panel (a) shows representative direct reactive trajectories, panel (b) roaming reactive, panel (c) direct nonreactive and panel (d) roaming nonreactive.

At this point, it is interesting to compare the present results on ketene isomerization with those obtained in our previous studies of the roaming phenomenon [14, 15], which were done in the context of an ion-molecule reaction. We see that in the present problem, the natural classification into four different categories of the trajectories fits nicely into the classification provided in the previous studies. Our earlier analyses of the roaming phenomenon pointed out that roaming is related to the appearance of trapped trajectories between two DSs in phase space. This led us to define a roaming region which is the region of phase space between these two DSs, where the roaming occurs. The scenario here for ketene isomerization is similar, and the trapping occurs between the two DSs located in the vicinity of $\mathrm{EP}_{1}$ and $\mathrm{EP}_{7}$. These two DSs define a region in phase space, which can be identified with the roaming region we defined in our previous work. Furthermore, we previously described how nonlinear resonances manifest by the presence of certain POs provide the basic mechanism to transfer energy from one DoF to another and enhance the trapping phenomenon in the roaming region. We shall see below that this ingredient of the roaming phenomenon is also present in ketene isomerization and takes also the form of resonant POs. Lastly, we noted in our earlier studies that our approach had strong similarities with Miller's unified approach to reaction mechanisms in the presence of complex formation [27]. Specifically, Miller considered how TST should be
Fig. 3 The four different categories of trajectories at energy $E=0.04 E_{h}$. a Direct reactive trajectories. b Roaming reactive trajectories. c Direct nonreactive trajectories. d Roaming nonreactive trajectories

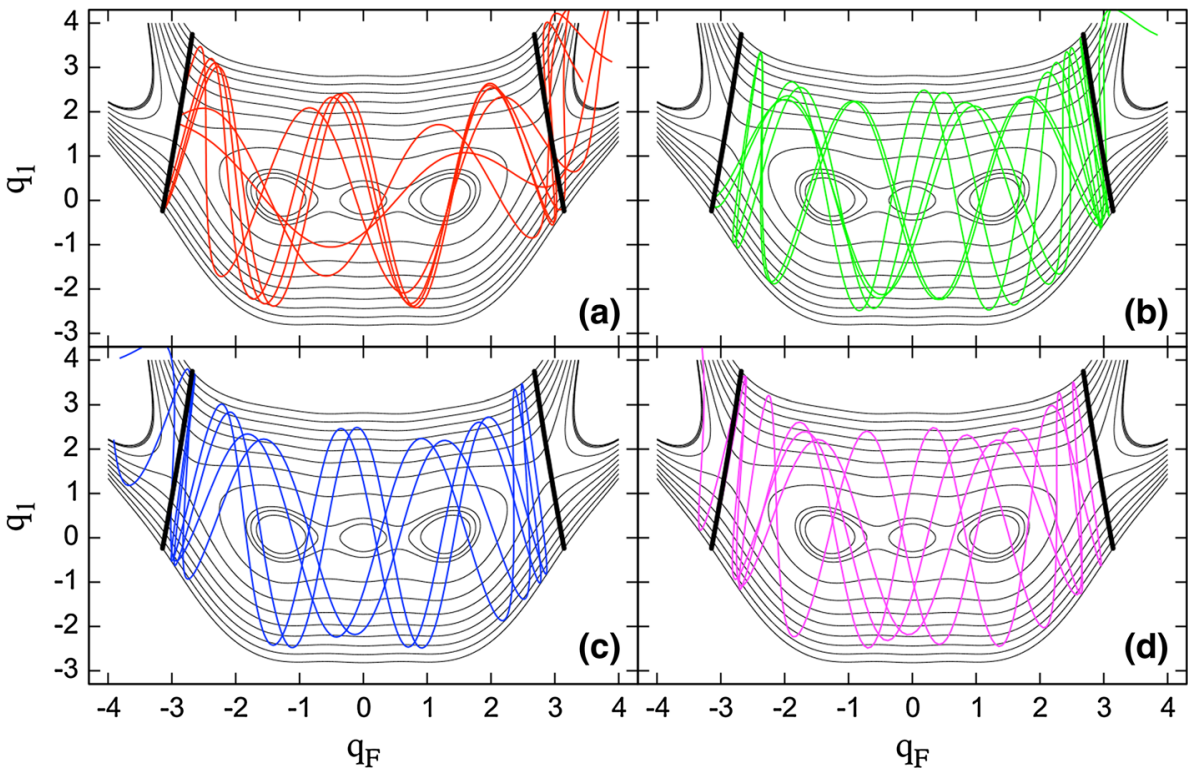


modified in the presence of a complex formation. This led us to investigate the question of the statistical assumption of TST in the roaming region using a gap time analysis, and we now investigate this question for the ketene isomerization.

\subsection{Gap time analysis}

The relevance of studying gap times in unimolecular reaction is seen in the investigation of the validity of the statistical assumption of TST. This assumption has been the subject of numerous works (see, for example the work of Slater [28, 29], Bunker [30, 31], Bunker and Hase [32], Thiele [33, 34], Dumont and Brumer [35] and DeLeon and co-workers [36, 37]).

In our earlier paper [15], we summarized the gap time approach to reaction rates due to Thiele [33, 34] (see also the discussion in Ref. [38]). The gap time in the ketene isomerization problem is the time $s$ it takes for a trajectory to traverse the roaming region. If we consider a point on the forward hemisphere of the DS located in the vicinity of $\mathrm{EP}_{1}$ (the hemisphere which intercepts trajectories travelling from the 'left' ketene well to the oxirene well), the gap time of a trajectory initiated at this point is the time it takes for this trajectory to reach either the hemisphere intercepting trajectories travelling from the oxirene well to the 'right' ketene well of the DS located in the vicinity of $\mathrm{EP}_{7}$ or to reach the backward hemisphere of the DS located in the vicinity of $\mathrm{EP}_{1}$ (the one which intercepts trajectories travelling from the oxirene well to the 'left' ketene well). An important notion in the gap time formulation of TST is the gap time distribution, $P(s ; E)$ : the probability that a phase space point on the forward hemisphere of the DS at $\mathrm{EP}_{1}$ at energy $E$ has a gap time between $s$ and $s+d s$ is equal to $P(s ; E) d s$. The statistical assumption of TST is equivalent to the requirement that the gap time distribution is the random, exponential distribution.

$P(s ; E)=k(E) \exp (-k(E) s)$.

This distribution is characterized by a single exponential decay constant $k(E)$, which is a function of the energy.

In addition to the gap time distribution, we also consider the integrated gap time distribution $F(t ; E)$, which is defined as the fraction of trajectories with gap times $s \geq t$, and is expressed as

$$
F(t ; E)=\int_{t}^{+\infty} \mathrm{d} s P(s ; E) .
$$

For the random gap time distribution, the integrated gap time distribution is exponential, $F(t ; E)=\exp (-k t)$.
The results of gap time and integrated gap time distributions for ketene isomerization are shown in Figs. 4 and 5. To obtain these figures, we sampled uniformly at a certain energy the forward hemisphere of the DS at $\mathrm{EP}_{1}$ and propagated trajectories until they exit the roaming region by either crossing the DS at $\mathrm{EP}_{7}$ or recrossed the DS at $\mathrm{EP}_{1}$. Figure 4 shows the gap time distributions at different energies measured from the oxirene minimum (see the caption of the figure). For each panel, we have reported the individual gap time distributions of the different categories of trajectories. The red curves represent the distributions for the direct reactive trajectories, the green represent the roaming reactive trajectories, the blue the direct nonreactive and the magenta the roaming nonreactive trajectories. The black curves depict the gap time distributions for all categories taken together. Figure 5 shows the integrated gap time distributions at the same energies as Fig. 4. For each energy, we show two panels presenting the short timescale distributions and the large timescale distributions. The different curves with their colours have the same meaning as in Fig. 4.

As we can see, these two figures exhibit significant deviation from the random gap time distributions as well as exponential integrated gap time distributions, indicating that the statistical assumption of TST is not valid for the ketene isomerization problem and must be corrected in some fashion. To give an idea of the kind of corrections one needs to provide, we plot in Fig. 6 the fractions of the different types of trajectories as a function of the energy. If one were to use the $\mathrm{DS}$ at $\mathrm{EP}_{1}$ to compute the flux needed to evaluate the reaction rate in TST, this flux would have to be corrected by the fractions given in Fig. 6. Indeed, since some trajectories are reflected back to the 'left' ketene well, there are some recrossing trajectories and the real flux is the flux through the DS multiplied by the sum of the fractions of the direct and roaming reactive trajectories.

\subsection{Trapping in the roaming region and resonant periodic orbits}

We have attributed the roaming phenomenon to trapped trajectories, which follow alternative pathways to those associated with MEPs on the PES. The trapping is due to multiple dividing surfaces and the existence of nonlinear mechanical resonances. Resonances can be traced in phase space by constructing continuation/bifurcation diagrams of periodic orbits starting from principal POs originating from equilibria, minima and saddles, and following them in a parameter space, which may include the total energy, angular momentum, masses or other parameters of interest. This is a well-known strategy in studying nonlinear dynamical systems.

For the ketene isomerization model, we located families of POs that are related to EP1 and EP7 equilibria and the 
Fig. 4 Gap time distributions For each energy, we give the number $n$ of trajectories used to compute the distributions. a Energy $E=0.010 E_{h}$, $n=942592$. b $E=0.0150 E_{h}$, $n=941938$. c $E=0.040 E_{h}$, $n=1967165$. d $E=0.150 E_{h}$, $n=1932961$
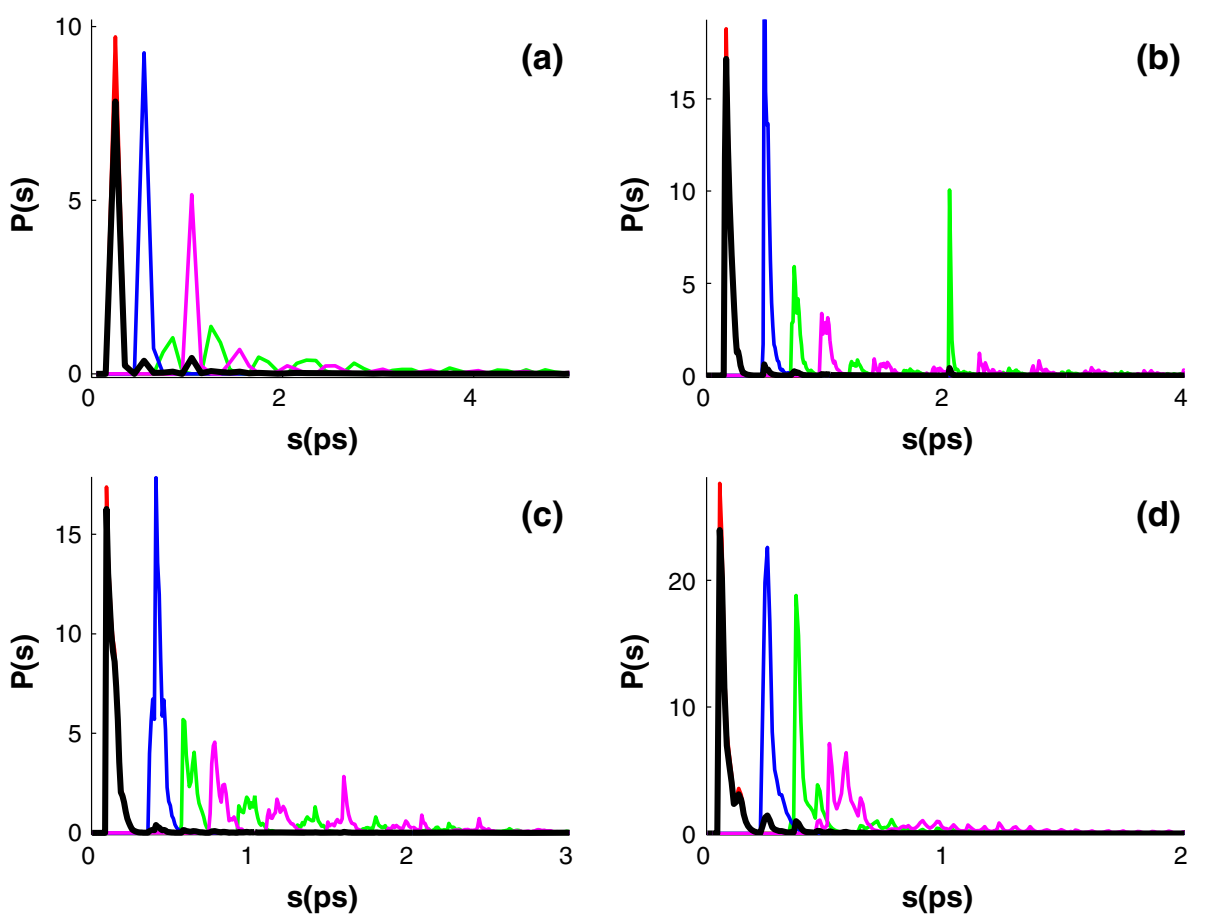

roaming region at low energies of excitation. These families emanate from a cascade of centre-saddle bifurcations approaching the saddle from energies above the isomerization threshold [39]. The results are shown in a continuation/bifurcation diagram of POs in Fig. 7. This figure depicts the period of the POs found as a function of energy. We also label the POs with respect to their resonance numbers $n: m$, which means that the PO makes $n$ oscillations in $q_{F}$ direction in the time it performs $m$ oscillations in $q_{1}$ direction. To give an idea of the shape of these POs in configuration space, we have plotted the 1:6 resonant $\mathrm{PO}$ as a blue line in Fig. 1. It is easy to recognize the impact of this family of POs on the dynamics of ketene from the plots of representative trajectories in Fig. 3. It is worth emphasizing that these periodic orbits are the results of centresaddle bifurcations. Thus, we expect, and indeed find, stable and unstable branches of POs to appear simultaneously, and these periodic orbits define the resonance region in phase space. A linear stability analysis of periodic orbits reveals the stable/unstable character of nearby trajectories with respect to POs. However, in order to better estimate the size of the resonance zone defined by these periodic orbits, we calculate Poincaré surfaces of section (PSS) [40], and autocorrelation functions from batches of trajectories sampled around the POs.

\subsubsection{Poincaré surfaces of section}

We have used two different PSS, which give complementary views of the dynamics. The first one $\left(\mathrm{PSS}_{1}\right)$ was defined by fixing $q_{F}=0$ and recording points when they cross this plane with a momentum $p_{q_{F}}>0$. The second one $\left(\mathrm{PSS}_{F}\right)$ was chosen at $q_{1}=0$ with momentum $p_{q_{1}}>0$. These two PSS are depicted in Fig. 8 for two different energies. Panel (a) is $\mathrm{PSS}_{1}$ for energy $E=0.01$ Hartree above oxirene minimum and panel (b) is $\mathrm{PSS}_{F}$ for the same energy. Panels (c) and (d) are the equivalent PSS at energy $E=0.04$ Hartree above oxirene minimum.

For each panel, we have used different colours for points on the PSSs belonging to trajectories of a different category as were classified above. The colours are consistent with those in the previous plots: hence, red dots denote points belonging to direct reactive trajectories, green dots denote the roaming reactive ones, blue the direct nonreactive and magenta the roaming nonreactive trajectories, respectively. In Fig. 8, we see a clear separation between the red and the other colours with the red concentrated in the middle of the figure. This is consistent with the fact that direct reactive trajectories have their energy mainly distributed on the $\left(q_{F}, p_{q_{F}}\right)$ DoF. Interestingly, the blue nonreactive direct trajectories appear at the boundary between the red and the roaming trajectories. Such reactivity boundaries have been observed in many studies and in several different contexts (see for example [15, 41-43], and references therein).

On the other hand, the green and magenta points (roaming trajectories) cover the periphery in the plots of $\mathrm{PSS}_{1}$ indicating that more energy is involved in the $\left(q_{1}, p_{q_{1}}\right)$ DoF for these types of trajectories. The two plots of $\mathrm{PSS}_{F}$ for the two energies show that the green and magenta dots are concentrated around some islands of 
Fig. 5 Lifetime distributions. For each energy, we plot two panels showing the small timescale and the large timescale of the distribution. The number of trajectories used to compute the distributions is as indicated in Fig. 4. a, b Energy $E=0.010 E_{h}$. c, d $E=0.0150 E_{h}$. e, f $E=0.040$ $E_{h} . \mathbf{g}, \mathbf{h}$ Energy $E=0.150 E_{h}$
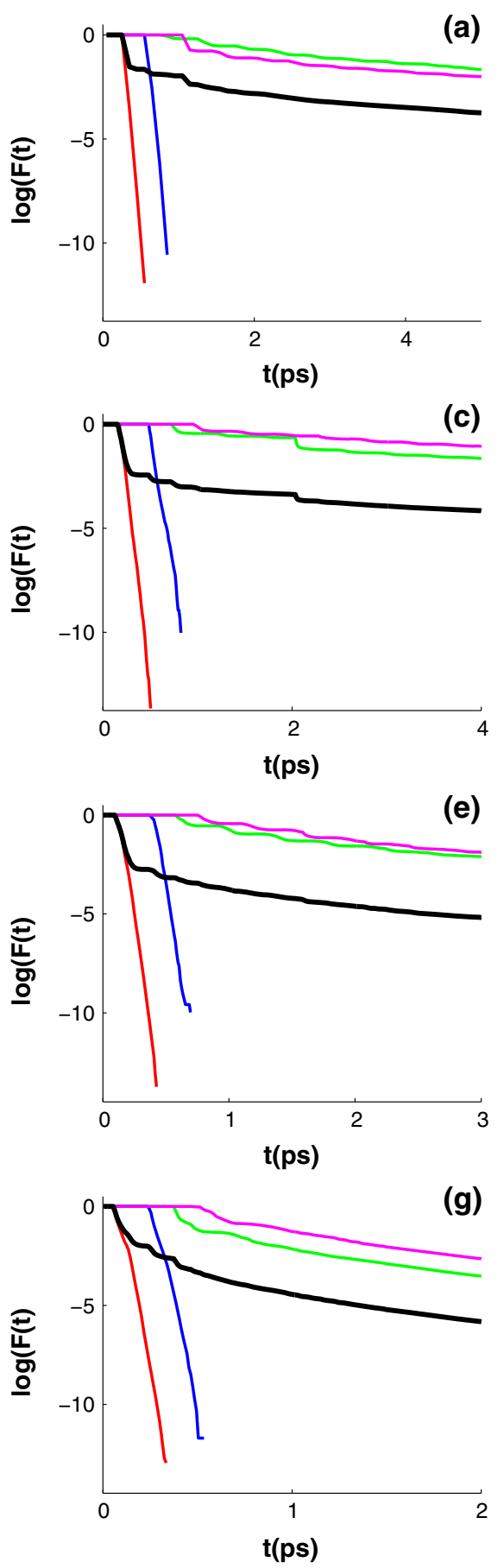
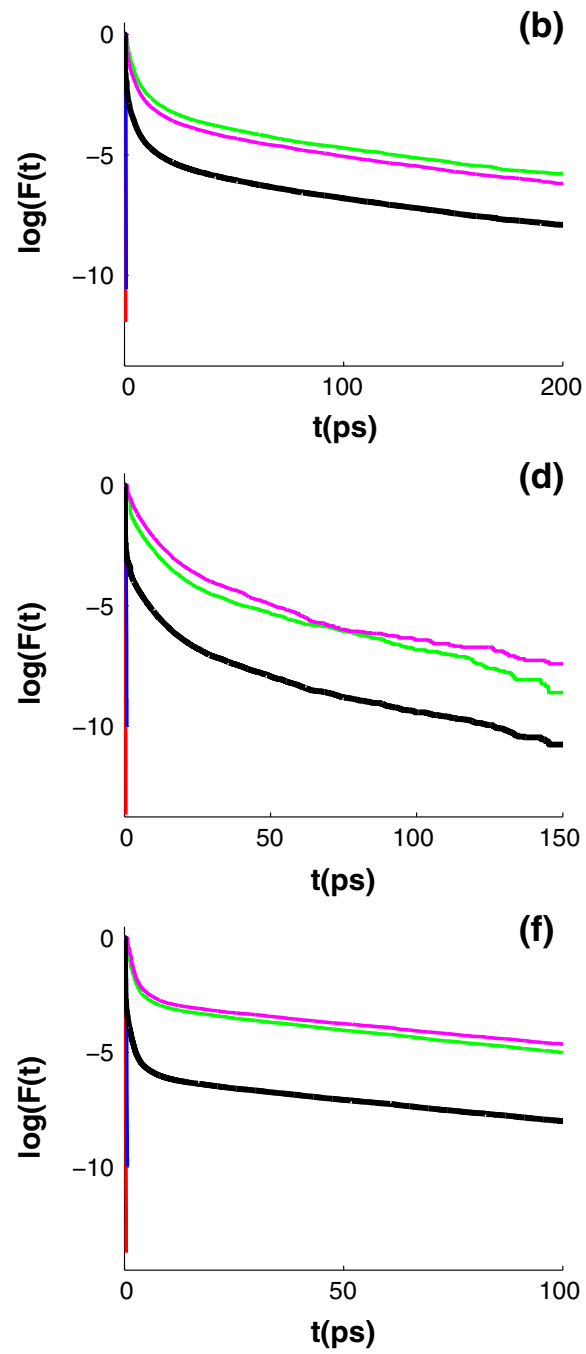

(b)

(d)

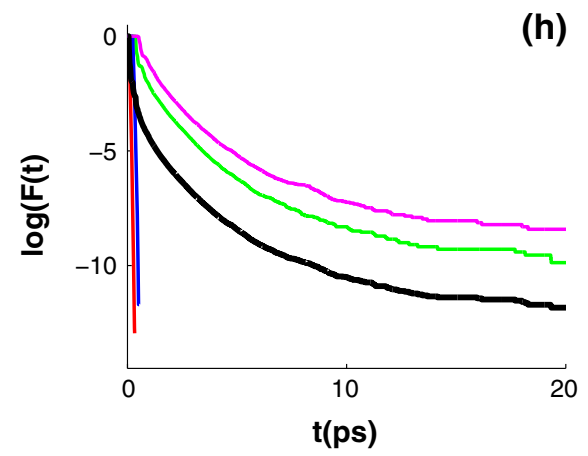

stability (blank area). These blank areas correspond to the stable regions in phase space associated with the stable resonant POs. The large blank area in panel (b) and for the energy $E=0.01$ Hartree is not accessible to trajectories initiated on the DS at EP 1 . However, as energy increases to 0.04 Hartree, this stable area shrinks and the three central blank domains in panel (d) are attributed to trajectories in the vicinity of the three minima, which correspond to oxirene $\left(\mathrm{EP}_{4}\right)$ and the two formylmethylene minima $\left(\mathrm{EP}_{2}, \mathrm{EP}_{6}\right)$.

\subsubsection{Classical autocorrelation functions}

Quantum calculations of the rate constant of ketene isomerization have shown that there are resonant features that are spaced at $70-80 \mathrm{~cm}^{-1}$ apart and with a width of about $10 \mathrm{~cm}^{-1}$. These resonances have been attributed to Feshbach energy transfer or dynamical resonances that occur at energies above the barrier to isomerization [13]. According to Gezelter and Miller [13], 'these dynamical resonances appear because the multimode potential energy surface has 

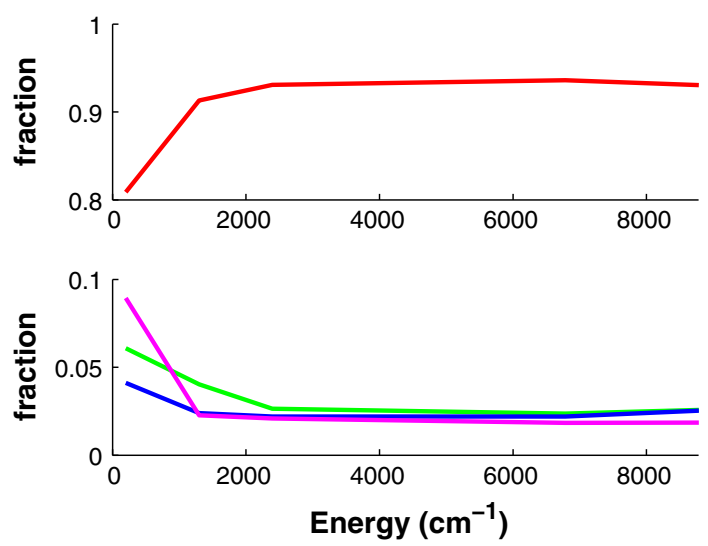

Fig. 6 Fractions of the different trajectories categories as a function of the energy above the barrier for isomerization. The different fractions are computed with the same number of trajectories as indicated in Fig. 4

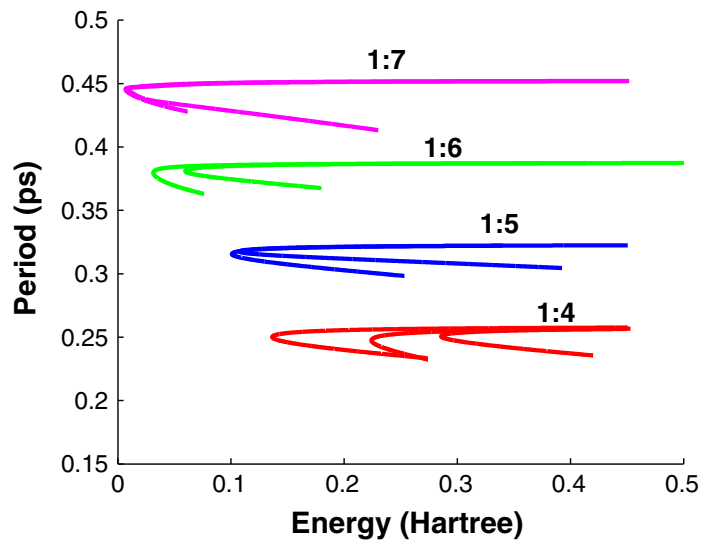

Fig. 7 Continuation/Bifurcation diagram for the resonant periodic orbits in the roaming region

a strongly bent region between the two outer transition states, which acts as a dynamical bottleneck even at total energies above the outer transition state energies'. The present classical trajectory results suggest that these resonances can be identified with the resonant periodic orbits, which are the result of a cascade of centre-saddle bifurcations shown in Fig. 7 [44].

This notion can be verified by calculating the classical analogue $(\Omega(t))$ of the quantum survival probability function $\left(|C(t)|^{2}\right)$ for a wavepacket centred on the classical PO. In this case, the correspondence between spectrum and phase space structure is relatively straightforward. We can pass from the quantum to the classical analogue of the autocorrelation function by replacing the trace in the density matrix given in Eq. 7 with an integral over the phase space, and by replacing the density operators $(|\phi(q, t)\rangle\langle\phi(q, t)|)$ with classical distribution functions, $\rho(q, p),[45,46]$.
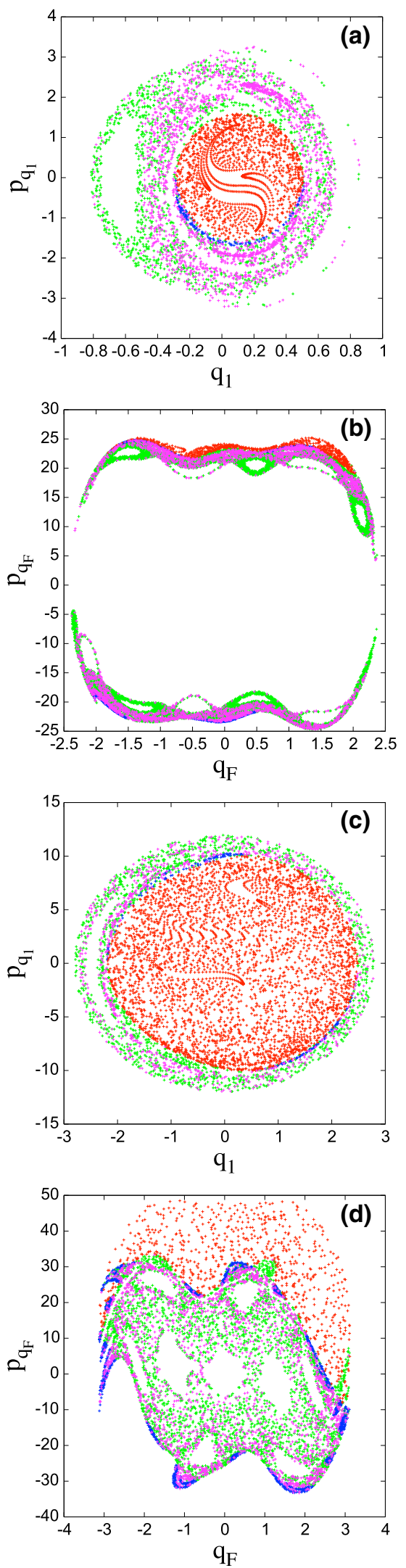

Fig. 8 Poincaré surfaces of sections (PSSs). a PSS ${ }_{1}$ at $E=0.010 E_{h}$. b $\mathrm{PSS}_{F}$ at $E=0.010 E_{h}$. c PSS 1 at $E=0.040 E_{h}$. d PSS $\mathrm{PS}_{F}$ at $E=$ $0.040 E_{h}$ 


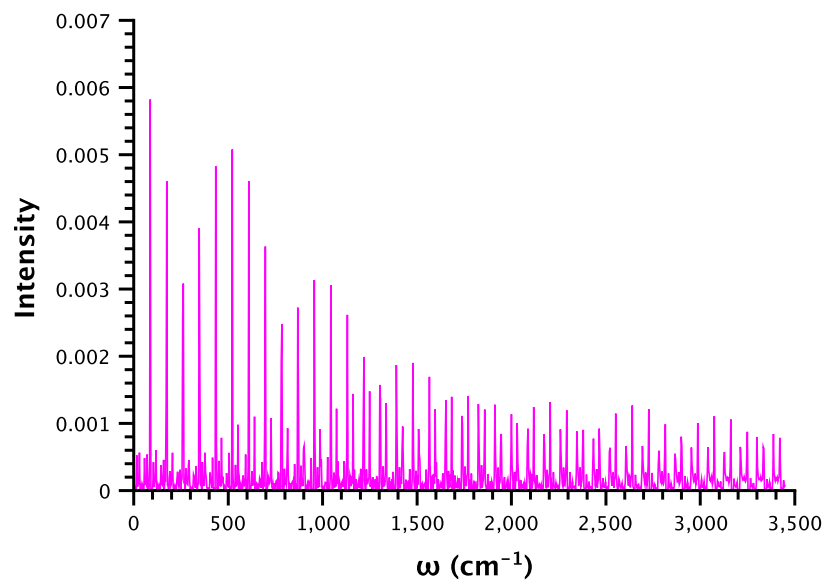

Fig. 9 Autocorrelation function for trajectories in the neighbourhood of 1:6 periodic orbit at energy of $0.04 E_{h}$

$$
\begin{aligned}
& \Omega(t)=\int \rho[q(0), p(0)] \rho[q(t), p(t)] \mathrm{d} q \mathrm{~d} p . \\
& \begin{aligned}
|C(t)|^{2} & =|\langle\phi(q, 0) \mid \phi(q, t)\rangle|^{2} \\
& =\langle\phi(q, 0) \mid \phi(q, t)\rangle\langle\phi(q, t) \mid \phi(q, 0)\rangle,
\end{aligned}
\end{aligned}
$$

In our case, the classical initial distribution $\rho[q(0), p(0)]$ is a Gaussian function of coordinates and momenta. The spectrum is then defined as the Fourier transform,

$I_{c}(\omega)=\int e^{i \omega t} \Omega(t) \mathrm{d} t$.

The classical survival probability function, Eq. 6, and its Fourier transform have been used in studies of molecular spectroscopy and dynamics by Gomez Llorente, Pollak and Taylor [47, 48].

Figure 9 shows a typical spectrum produced by a batch of 1000 trajectories selected from a Gaussian distribution centred on the stable 1:6 PO and at energy $0.04 E_{h}$. The spacing of eigenfrequencies is $83 \mathrm{~cm}^{-1}$ and the regularity of the spectrum reflects the regularity of the region of phase space in which the trajectories are trapped. Higher-order POs, like the 1:7, have longer periods and appear at energies closer to the isomerization saddle. Their frequencies match the quantum mechanical frequencies in the interval of $70-80 \mathrm{~cm}^{-1}$ found by Gezelter and Miller [13].

\section{Phase space reaction pathways and roaming}

Reaction pathways are conventionally identified in nuclear configuration space by minimum energy paths on the potential energy surface, since the latter is the fundamental concept used to interpret chemical reactivity [26]. Thus, the presence of multiple saddles on the PES suggests the existence of multiple reaction pathways. However, the development of nonlinear mechanics has enabled the rigorous definition of objects such as reaction pathways and transition states in phase space for polyatomic molecules.

The strategy followed to understand the motions of nonlinear dynamical systems is to reveal the geometry of phase space in a systematic way by first locating equilibria, and from them the emanating principal families of periodic orbits, tori, NHIMs and (un)stable manifolds associated with NHIMs. The latter are key protagonists in controlling reaction fluxes. Both for the isomerization dynamics of ketene and in our previous work on the Chesnavich model for ion-molecule reactions, we have shown that, by defining transition states using the appropriate NHIMs (unstable periodic orbits for 2D molecular models), ordinary (MEP) and roaming reaction pathways emerge within a phase space framework. Similarities between the two type of reactions emphasized in Sect. 3.2; we now point out some significant differences.

ketene belongs to the general type of molecules for which NHIMs are associated with potential energy saddles. The Chesnavich model for ion-molecule reactions is a model, which manifests the existence of NHIMs not associated with potential energy saddles. Indeed, as shown in refs $[14,15]$, the loose transition state and the tight one are periodic orbits originating from centre-saddle bifurcations. The loose transition state is a rotating-type periodic orbit (relative equilibrium). The tight transition state is a periodic orbit associated with the barrierless MEP for association/dissociation. Hence, for these type of reactions, phase space is mandatory to define reaction pathways, since the potential energy alone is inadequate.

By attributing roaming to trapped trajectories in the region of nonlinear resonances in phase space, we are able to generalize the concept of roaming. From the PSS in Fig. 8 and in the low energy regime ( 0.01 Hartree), the roaming pathways are determined by the resonances 1:6 and 1:7 as indicated by the types of POs in Fig. 7. From the large number of trajectories run, none penetrates close to the regions of formylmethylene and oxirene. At higher energies (0.04 Hartree), the regular phase space region governed by the equilibria, EP2, EP4 and EP6, shrinks, and the reactive trajectories initiated from the NHIMs of the outer saddle penetrate into this region. In this way, we infer that the new resonances associated with these minima define new roaming reaction pathways. It is worth mentioning that nonlinear resonances associated with centre-saddle bifurcations of periodic orbits have been found to be a general phenomenon in molecular dynamics [44].

Finally, it is essential to stress that, although roaming as an alternative reaction pathway was identified in the dissociation of formaldehyde with long-range interactions between the fragment species, several other molecules with 
competing non-MEP have previously been studied [49-55].

\section{Summary and conclusions}

In this paper, we have used a reduced dimensional model initially proposed by Gezelter and Miller to study the isomerization dynamics of ketene molecule. The study of Ulusoy et al. [6, 7] discussed the connection of ketene isomerization and roaming reactions mechanism. The question of how our previous interpretation of roaming phenomenon $[14,15]$ fits the ketene isomerization situation naturally arose.

It was found that the previous interpretation of the roaming mechanism in terms of a trapping mechanism of trajectories between two DSs enhanced by nonlinear resonances also fits the ketene isomerization reaction well. We were able to classify classical trajectories into qualitatively different types of trajectories and compute fractions of different types of trajectories; these data could be used to correct the flux in a reaction rate calculation. As in our previous study, the question of statistical dynamics was raised and investigated by gap time analysis. Significant deviation from the statistical assumption of TST was found. In addition, evidence of the trapping mechanism of the trajectories by resonant POs has been given using surfaces of section.

The importance of ketene isomerization stems from the experimental investigations of Moore and co-workers. Furthermore, the quantum mechanical results of Gezelter and Miller are in overall agreement to the experimental results. Our phase space analysis provides further insight into this important system; for example, ketene provides an experimental manifestation of the importance of the centresaddle bifurcations of periodic orbits.

In the present calculation and for the energies studied, the direct-type reactive trajectories dominate (Fig. 6). However, if oxirene could be isolated and photoexcited into resonant states, the roaming branch of the reaction would then be the dominant one. In other words, knowledge of the phase space structure in a particular range of energies in principle enables us to exert some degree of control over chemical reaction pathways. Analysis of classical phase space as presented here can achieve a degree of resolution not necessarily accessible to experiment. Nevertheless, experimental techniques are continually advancing, and methods may be developed in the future to control roaming pathways [56].

Acknowledgments We wish to thank Dr. D. Gezelter, Prof. W. H. Miller and Prof. R. Hernandez and coworkers for helpful discussions. This work is supported by the National Science Foundation under
Grant No. CHE-1223754 (to GSE). FM, PC and SW acknowledge the support of the Office of Naval Research (Grant No. N00014-01-10769), the Leverhulme Trust, and the Engineering and Physical Sciences Research Council (Grant No. EP/K000489/1).

Open Access This article is distributed under the terms of the Creative Commons Attribution License which permits any use, distribution, and reproduction in any medium, provided the original author(s) and the source are credited.

\section{References}

1. Lovejoy ER, Kim SK, Alvarez RA, Moore CB (1991) J Chem Phys 95:4081

2. Lovejoy ER, Kim SK, Moore CB (1992) Science 256(5063):1541

3. Lovejoy ER, Moore CB (1993) J Chem Phys 98:7846

4. Kirmse W (2002) Eur J Org Chem 2002(14):2193

5. Scott AP, Nobes RH, Schaefer HF III, Radom L (1994) J Am Chem Soc 116(22):10159

6. Ulusoy IS, Stanton JF, Hernandez R (2013) J Phys Chem A 117:7553

7. Ulusoy IS, Stanton JF, Hernandez R (2013) J Phys Chem A 117:10567

8. Townsend D, Lahankar SA, Lee SK, Chambreau SD, Suits AG, Zhang X, Rheinecker J, Harding LB, Bowman JM (2004) Science 306(5699): 1158

9. van Zee RD, Foltz MF, Moore CB (1993) J Chem Phys 99:1664

10. Yu HG (2011) Physica Scripta 84(2):028104

11. Bowman JM, Shepler BC (2011) Ann Rev Phys Chem 62:531

12. Bowman JM (2014) Mol Phys. doi:10.1080/00268976.2014. 897395

13. Gezelter JD, Miller WH (1995) J Chem Phys 103:7868

14. Mauguière FAL, Collins P, Ezra GS, Farantos SC, Wiggins S (2014) Chem Phys Lett 592:282

15. Mauguière FAL, Collins P, Ezra GS, Farantos SC, Wiggins S (2014) J Chem Phys 140:134112

16. Chesnavich WJ (1986) J Chem Phys 84:2615

17. Gezelter D Private communication

18. Baer T, Hase WL (1996) Unimolecular reaction dynamics. Oxford University Press, New York

19. Pechukas P, McLafferty FJ (1973) J Chem Phys 58(4):1622

20. Pechukas P, Pollak E (1977) J Chem Phys 67(12):5976

21. Pollak E, Pechukas P (1978) J Chem Phys 69:1218

22. Pechukas P, Pollak E (1979) J Chem Phys 71(5):2062

23. Waalkens H, Wiggins S (2004) J Phys A Math Gen 37(35):L435

24. Moser J (1976) Commun Pure Appl Math 29:727

25. Kelley A (1969) Pac J Math 29(2):335

26. Heidrich D (1995) The reaction path in chemistry: current approaches and perspectives. Kluwer, Boston

27. Miller WH (1976) J Chem Phys 65:2216

28. Slater NB (1956) J Chem Phys 24(6): 1256

29. Slater NB (1959) Theory of unimolecular reactions. Cornell University Press, Ithaca

30. Bunker DL (1962) J Chem Phys 37:393

31. Bunker DL (1964) J Chem Phys 40:1946

32. Bunker DL, Hase WL (1973) J Chem Phys 59:4621

33. Thiele E (1962) J Chem Phys 36(6):1466

34. Thiele E (1963) J Chem Phys 38(8):1959

35. Dumont RS, Brumer P (1986) J Phys Chem 90:3509

36. DeLeon N, Berne BJ (1981) J Chem Phys 75:3495

37. Berne BJ, DeLeon N, Rosenberg RO (1982) J Phys Chem 86:2166

38. Ezra GS, Waalkens H, Wiggins S (2009) J Chem Phys 130:164118 
39. Lin SY, Guo H, Farantos SC (2005) J Chem Phys 122(12): 124308

40. Wiggins S (2003) Introduction to applied nonlinear dynamical systems and chaos, 2nd edn. Springer, New York

41. Nagahata Y, Teramoto H, Li C, Kawai S, Komatsuzaki T (2013) Phys Rev E 88:042923

42. Grice M, Andrews B, Chesnavich W (1987) J Chem Phys 87:959

43. Mauguiere F, Collins P, Ezra G, Wiggins S (2013) J Chem Phys 138:134118

44. Farantos SC, Schinke R, Guo H, Joyeux M (2009) Chem Rev 109(9):4248

45. Baranger M (1958) Phys Rev A 111:481

46. Heller EJ, Davis MJ (1980) J Phys Chem 84:1999

47. Gomez Llorente JM, Pollak E (1989) J Chem Phys 90:5406

48. Gomez Llorente JM, Taylor HS (1989) J Chem Phys 91:953
49. Hu X, Hase WL (1989) J Phys Chem 93:6029

50. Sun L, Hase WL (2002) Science 296:875

51. López JG, Vayner G, Lourderaj U, Addepalli SV, Kato S, deJong WA, Windus TL, Hase WL (2007) J Am Chem Soc 129:9976

52. Mikosch J, Trippel S, Eichhorn C, Otto R, Lourderaj U, Zhang JX, Hase WL, Weidemüller M, Wester R (2008) Science 319:183

53. Zhang J, Mikosch J, Trippel S, Otto R, Weidemüller M, Wester R, Hase WL (2010) J Phys Chem Lett 1:2747

54. Carpenter BK (2004) In Moss RA, Platz MS, Jones M Jr (eds) Reactive intermediate chemistry. Wiley, New York, pp 925-960

55. Lourderaj U, Hase WL (2009) J Phys Chem A 113:2236

56. Ashfold MNR, Nahler N, Orr-Ewing A, Vieuxmaire O, Toomes R, Kitsopoulos T, Garcia I, Chestakov D, Wu S, Parker D (2006) Phys Chem Chem Phys 8:26 